\title{
Phenotypic and Genetic Relationships between Kidney Function and Carotid Intima-Media Thickness in Koreans: The Healthy Twin Study
}

\author{
Kayoung Lee $^{\mathrm{a}}$ Joohon Sung ${ }^{\mathrm{b}}$ Sang Cheol Lee ${ }^{c}$ Seung Woo Park \\ Youn Sic Kim $^{b}$ Joo Yeon Lee ${ }^{b}$ Yun-Mi Song ${ }^{d}$ \\ ${ }^{a}$ Department of Family Medicine, Busan Paik Hospital, Inje University College of Medicine, ${ }^{b}$ Department of \\ Epidemiology, School of Public Health and Institute of Health and Environment, Seoul National University, \\ 'Department of Cardiology and Cardiovascular Imaging Center, Cardiac and Vascular Center, Samsung Medical \\ Center, and d Department of Family Medicine, Samsung Medical Center and Center for Clinical Research, \\ Samsung Biomedical Research Institute, Sungkyunkwan University School of Medicine, Seoul, Korea
}

\section{Key Words}

Albuminuria - Carotid atherosclerosis - Genetic variation • Glomerular filtration rate $\cdot$ Risk factor

\begin{abstract}
Background: The phenotypic and genetic relationships between carotid intima-media thickness (CIMT) and estimated glomerular filtration rate (eGFR) or urinary albumin-creatinine ratio (ACR) were evaluated in Korean twins and families. Methods: We recruited 688 participants (296 individual twins and 392 singletons, age 30-74 years) who were without myocardial infarction and stroke among participants in the Healthy Twin Study. eGFR was calculated using the Chronic Kidney Disease Epidemiology Collaboration equation. CIMT was measured using B-mode carotid ultrasound. Covariates were sex, Framingham risk scores, alcohol use, exercise, BMI, high-sensitivity C-reactive protein and triglycerides. Quantitative genetic and linear mixed analyses were performed. Results: In adjusted models there were associations between kidney function and CIMT with [beta] estimates ranging from -0.022 to -0.032 between eGFR and CIMT, and from $0.042-0.060$ between ACR and CIMT. The covariate-adjusted heritabilities for eGFR, ACR and composite CIMT were $0.75,0.32$ and 0.45 , respectively $(p<0.001)$. The
\end{abstract}

adjusted genetic correlation between eGFR and CIMT was from -0.20 to $-0.28(p<0.05)$, whereas there was no genetic correlation between ACR and CIMT. Conclusion: Kidney function is a surrogate marker of carotid atherosclerosis and further studies of the pleiotropic relationships between CIMT and eGFR are warranted.

Copyright $\odot 2012$ S. Karger AG, Basel

\section{Introduction}

Chronic kidney disease (CKD) is closely associated with cardiovascular disease (CVD). In a clinical setting CKD can be evaluated by the estimated glomerular filtration rate (eGFR) and by the assessment of albuminuria as the ratio of albumin to creatinine in urine (ACR) [1]. Carotid intima-media thickness (CIMT) is positively associated with risk of CVD and is a sensitive, reproducible surrogate to determine the presence of subclinical atherosclerosis [2]. In this regard, the relationships between eGFR or ACR and CIMT have been evaluated prior to the overt development of CKD and CVD in order to better understand the underlying mechanisms linking the two conditions. However, the relationships between CIMT and eGFR or ACR are inconsistent between studies [3-7], probably due

\section{KARGER}

Fax +4161306 1234

E-Mail karger@karger.ch

www.karger.com
(C) 2012 S. Karger AG, Basel

$1420-4096 / 12 / 0354-0259 \$ 38.00 / 0$

Accessible online at:

www.karger.com/kbr
Prof. Yun-Mi Song, MD, MPH, PhD, Department of Family Medicine

Samsung Medical Center and Center for Clinical Research

Samsung Biomedical Research Institute, Sungkyunkwan University School of Medicine 50 Ilwondong, Kangnamgu, Seoul 135-710 (Korea)

Tel. +82 23410 2442, E-Mail yunmisong@ skku.edu 
to differences in adjustment for cardiovascular (CV) risk factors and in the demographic and clinical characteristics of study subjects with respect to sex, age, presence of diabetes, hypertension and chronic renal failure. Therefore, further examinations of whether reduced eGFR and increased ACR are independently associated with CIMT after adjustment for probable CV risk factors may be warranted. One study showed that Asians with low eGFR were at higher risk of stroke than other populations [8], suggesting that population origin affects the relationship between eGFR and CVD and emphasizing the need for further investigations of genetic or environmental factors that may affect these traits in Asian populations.

The aim of the present study was to examine whether there are independent associations between eGFR, ACR and CIMT, and whether there are genetic and/or environmental correlations between these traits in Korean twins and their family members after adjustment for a wide range of CV risk factors. Although both kidney function and CIMT measures are known to be under genetic control [9-14], to our knowledge the possible genetic correlation between kidney function and CIMT has never been studied.

\section{Methods}

\section{Study Sample}

The current study included 688 individuals (244 males and 444 females) from the Healthy Twin Study: 296 twins (238 individual monozygotic twins and 58 individual dizygotic twins) and 392 singletons from 196 families. The Healthy Twin Study has been part of the Korean Genomic Epidemiologic Study since 2005 and the design and detailed methods of the study have been published previously [15]. The zygosity of each twin pair was tested by the AmpFlSTR Identifier Kit (PerkinElmer, Waltham, Mass., USA) using 16 short tandem repeat (STR) markers (15 autosomal STR markers and one sex-determining marker) in $67 \%$ of the participating twin pairs. For the remaining $33 \%$, a zygosity-determining questionnaire with validated accuracy $>90 \%$ was applied [16].

All the participants were between 30 and 74 years of age in order to apply the age classification scheme of the Framingham Risk Scores (FRS) [17]. None had previous myocardial infarction and all had undergone evaluations for CV risk factors and CIMT measurement. Written informed consent was obtained from each participant. All study procedures were approved by the institutional review boards of the participating institutions.

\section{Clinical and Biochemical Measurements}

A self-administered standard questionnaire was used to collect information about demographic characteristics, alcohol consumption, cigarette smoking, physical exercise of more than moderate intensity, and medical history of hypertension and diabetes.
Blood pressure was measured using a manual sphygmomanometer and BMI was calculated. All biochemical analyses were conducted in a central laboratory. Overnight fasting venous blood samples were drawn to measure plasma glucose, serum lipid profile, high-sensitivity C-reactive protein and creatinine. An overnight 12-hour urine sample was collected to measure urinary albumin and creatinine. Serum and urinary creatinine were analyzed by Jaffe's photometric method (Cobas Mira, Roche, Basel, Switzerland) and urinary albumin was assessed by a colorimetric method (Cobas Mira). eGFR was calculated using the Chronic Kidney Disease Epidemiology Collaboration equation [18]. Urinary ACR was calculated by dividing the urinary albumin concentration by the urinary creatinine concentration [1]. The FRS for predicting coronary artery disease risk was determined by calculating the score of the Framingham points assigned to each risk factor, such as age, low- and high-density lipoprotein cholesterol, blood pressure, cigarette smoking and diabetes mellitus [17].

CIMT measurements were performed according to standardized protocols during the end-diastolic phase between the $\mathrm{P}$ and $Q$ wave of the electrocardiogram trace, using the automated IMT package and a high-resolution B-mode ultrasound (VIVID, General Electric, Horten, Norway or EKO7, Medison, Cypress, Calif., USA) equipped with a 7-MHz linear transducer. CIMT scans included the far walls of three segments, defined as follows: 10-20 $\mathrm{mm}$ proximal to the tip of the flow divider into the common carotid artery (CCA); the carotid bifurcation beginning at the tip of the flow divider and extending $10 \mathrm{~mm}$ proximal to the flow divider tip (Bif), and the proximal $10 \mathrm{~mm}$ of the internal carotid artery (ICA). Composite CIMT was calculated as the mean of the values for the three carotid artery segments of both sides. We estimated the reliability of IMT measurement in 14 randomly chosen participants whom IMT was repeatedly measured using the two different carotid ultrasound machines (VIVID and EKO7). The intraclass correlation coefficients for CCA, Bif and ICA were $0.93,0.86$, and 0.90 , respectively.

\section{Statistical Analysis}

log transformations were applied to CIMT measurements to approximate normal distributions. Linear trends in the distributions of CIMT values and CV risk factors according to the tertile distributions of eGFR or ACR were evaluated using linear regression analysis or $\chi^{2}$ tests. Relationships between kidney function and CIMT values were assessed using linear mixed models in which random effects (household and sibling effects) and fixed effects (sex, FRS, study site, alcohol consumption, exercise, BMI, triglycerides, high-sensitivity C-reactive protein, eGFR, and ACR) were considered. The linear trends of composite intimamedia thickening for subgroups divided by tertiles of eGFR (or ACR) and tertiles of the FRS after adjusting for other CV risk factors and ACR (or eGFR) were analyzed by linear mixed models. These analyses were performed using PASW Statistics 18 (Release 18.0.0 July 30, 2009, SPSS Inc., Chicago, Ill., USA).

Heritability, or the ratio of the additive variance component to the total trait variance, was estimated for eGFR, ACR, and IMT after adjusting for the covariates of sex, FRS, study site, alcohol consumption, exercise, BMI, triglycerides and high-sensitivity Creactive protein. To evaluate the presence of genetic and/or environmental regulation common to kidney function and CIMT values, we conducted bivariate analyses in which phenotypic correlations between the two traits in a single subject were partitioned 
Table 1. The associations of CIMT with eGFR and urinary ACR $(\mathrm{n}=688)$

\begin{tabular}{|c|c|c|}
\hline & \multicolumn{2}{|l|}{ CIMT } \\
\hline & $\begin{array}{l}\text { eGFR } \\
10 \mathrm{ml} / \mathrm{min} / 1.73 \mathrm{~m}^{2} \\
\beta \text {-estimate }(95 \% \mathrm{CI})\end{array}$ & $\begin{array}{l}\text { ACR } \\
50 \mathrm{mg} / \mathrm{g} \\
\beta \text {-estimate }(95 \% \mathrm{CI})\end{array}$ \\
\hline \multicolumn{3}{|l|}{ Composite } \\
\hline Crude & $-0.067(-0.082,-0.052)$ & $0.044(0.026,0.062)$ \\
\hline Adjusted & $-0.031(-0.045,-0.016)$ & $0.027(0.011,0.042)$ \\
\hline \multicolumn{3}{|c|}{ Common carotid artery } \\
\hline Crude & $-0.069(-0.084,-0.054)$ & $0.039(0.021,0.057)$ \\
\hline Adjusted & $-0.032(-0.047,-0.017)$ & $0.024(0.008,0.039)$ \\
\hline \multicolumn{3}{|c|}{ Carotid bifurcation } \\
\hline Crude & $-0.074(-0.091,-0.056)$ & $0.055(0.033,0.077)$ \\
\hline Adjusted & $-0.031(-0.049,-0.013)$ & $0.034(0.014,0.053)$ \\
\hline \multicolumn{3}{|c|}{ Internal carotid artery } \\
\hline Crude & $-0.051(-0.066,-0.035)$ & $0.018(-0.001,0.036)$ \\
\hline Adjusted & $-0.022(-0.038,-0.006)$ & $0.009(-0.008,0.027)$ \\
\hline \multicolumn{3}{|c|}{$\begin{array}{l}\text { Associations were assessed by linear mixed models including } \\
\text { random effects (household and sibling relation) and fixed effects. } \\
\text { Crude results include covariates of study site and eGFR (or ACR). } \\
\text { Adjusted results include covariates of sex, study site, BMI, alcohol } \\
\text { use, exercise, high-sensitivity C-reactive protein, triglycerides, } \\
\text { FRS, eGFR and ACR. }\end{array}$} \\
\hline
\end{tabular}

into genetic $\left(\rho_{\mathrm{G}}\right)$ and environmental correlations $\left(\rho_{\mathrm{E}}\right)$ with adjustment for the covariates. Significant deviation of $\rho_{\mathrm{G}}$ or $\rho_{\mathrm{E}}$ from zero was regarded as evidence of common sharing between the two traits in the same person. The Sequential Oligogenic Linkage Analysis Routines package (http://solar.sfbrgenetics.org; Version 4.2.0) was used to fit the data to a variance components model.

\section{Results}

Subjects with lower eGFR and higher ACR were more likely to be older, have greater carotid IMT, worse FRS, lipid profiles and higher fasting glucose levels. The prevalence of hypertension and BMI values tended to be higher and alcohol use was less prevalent among subjects with lower eGFR and higher ACR. The results of linear mixed analysis showed that decreased eGFR was independently associated with increased CIMT after adjusting for CV risk factors and ACR, and that the magnitudes of associations were similar across the IMT at each segment of the carotid artery. Increased ACR also had independent associations with increased IMT at CCA and Bif after adjusting for CV risk factors and eGFR (table 1). The combined effect of kidney function and CV risk on CIMT is

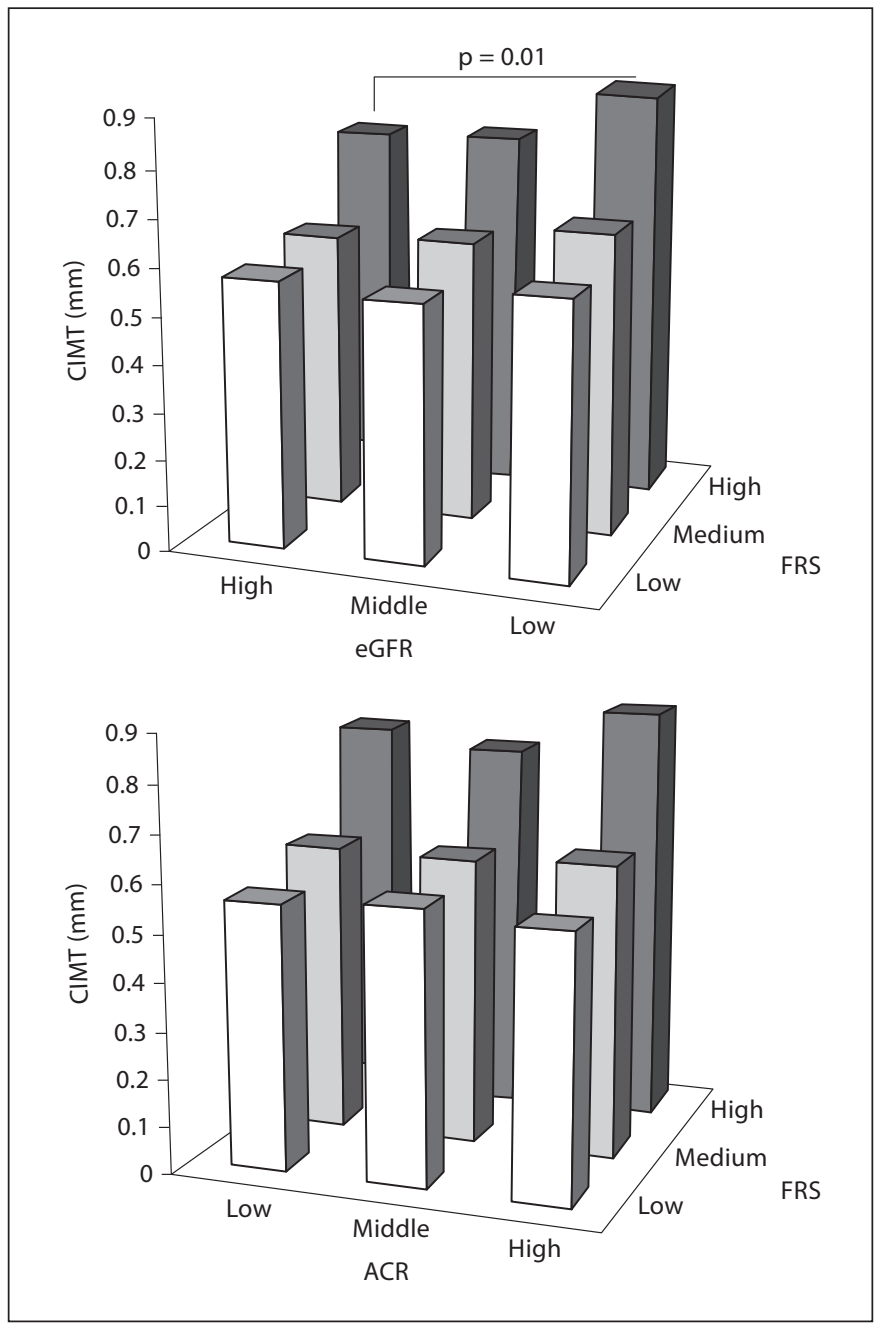

Fig. 1. The composite CIMT among the subgroups divided by tertiles of eGFR and the FRS (upper figure) or the subgroups divided by tertiles of ACR and the FRS (lower figure). The IMT decreased with increasing tertiles of eGFR in the third tertile of the FRS in the linear mixed models including random effects (household and sibling relation) and fixed effects (sex, study site, alcohol use, exercise, BMI, high-sensitivity C-reactive protein, triglycerides, ACR, and tertiles of eGFR; $\mathrm{p}=0.01$ ).

presented in figure 1 . The composite IMT of the carotid artery decreased with increasing tertiles of eGFR in the third tertile of the FRS after adjusting for other CV risk factors and ACR ( $p=0.01)$, while there were no significant linear trends for tertiles of eGFR in the lower two tertiles of the FRS. There were no increasing trends in CIMT for tertiles of the ACR in each tertile of the FRS. The adjusted heritability of eGFR was more than twice the heritability of ACR $(0.75 \pm 0.03,0.32 \pm 0.06, \mathrm{p}<$ 
Table 2. The cross-trait correlations between eGFR, urinary ACR and CIMT in the Healthy Twin Study $(\mathrm{n}=688)$

\begin{tabular}{|c|c|c|c|c|c|c|c|}
\hline & & \multicolumn{6}{|l|}{ CIMT } \\
\hline & & \multicolumn{3}{|l|}{ eGFR } & \multicolumn{3}{|l|}{ urinary ACR } \\
\hline Composite & crude & $-0.36^{*}$ & $-0.08(0.16)$ & $-0.56(0.05)^{*}$ & $0.15^{*}$ & $0.03(0.21)$ & $0.20(0.06)^{*}$ \\
\hline Common carotid & adjusted $^{\mathrm{a}}$ & $-0.17^{*}$ & $-0.28(0.08)^{*}$ & $-0.05(0.08)$ & $0.10^{*}$ & $0.12(0.14)$ & $0.09(0.07)$ \\
\hline \multirow[t]{2}{*}{ Carotid bifurcation } & crude & $-0.31^{*}$ & $-0.09(0.16)$ & $-0.50(0.06)^{*}$ & $0.13^{*}$ & $0.09(0.21)$ & $0.19(0.07)^{*}$ \\
\hline & adjusted $^{\mathrm{a}}$ & $-0.14^{*}$ & $-0.20(0.10)^{*}$ & $-0.09(0.08)$ & $0.12^{*}$ & $0.23(0.16)$ & $0.05(0.07)$ \\
\hline \multirow[t]{2}{*}{ Internal carotid } & crude & $-0.25^{*}$ & $-0.02(0.13)$ & $-0.47(0.06)^{*}$ & $0.09^{*}$ & $0.15(0.16)$ & $0.08(0.07)$ \\
\hline & adjusted $^{\mathrm{a}}$ & $-0.10^{*}$ & $-0.08(0.09)$ & $-0.15(0.08)^{*}$ & 0.03 & $0.08(0.09)$ & $-0.15(0.08)^{*}$ \\
\hline
\end{tabular}

${ }^{*} \mathrm{p}<0.05 . \rho_{\mathrm{G}}=$ Correlation explained by genetic share; $\rho_{\mathrm{E}}=$ correlation explained by nongenetic share. Adjusted results include covariates of sex, study site, BMI, alcohol use, exercise, high-sensitivity C-reactive protein, triglycerides and the FRS.

0.001 , respectively). Among carotid segments, heritability of IMT at the CCA was the highest $(0.47 \pm 0.05$, p $<$ $0.001)$, followed by IMT at the ICA and Bif $(0.45 \pm 0.05$, $0.36 \pm 0.06, \mathrm{p}<0.001$, respectively). Table 2 shows that eGFR had phenotypic correlations with IMT at the CCA even after adjusting for covariates, and that the correlations were explained by genetic cross-trait correlations between the two traits. By comparison, there were no genetic or environmental correlations between ACR and CIMT after adjusting for covariates.

\section{Discussion}

In this Korean twin and family study, CIMT showed an inverse association with eGFR after adjusting for CV risk factors and ACR, and the linear trend between tertiles of eGFR and CIMT was more likely to be distinct in the subgroup with higher CV risk. ACR was also positively associated with CIMT after adjusting for CV risk factors and eGFR, even though there were no significant linear trends between these traits among subgroups of the FRS. In bivariate genetic analysis, we found a crosstrait genetic correlation between eGFR and CCA-IMT, while there was no genetic correlation between ACR and CIMT. The genetic or environmental cross-trait correlations were consistent when the analyses were restricted for identical twins. Although reduced eGFR is an established risk factor for CVD, studies focusing on the rela- tionship between eGFR and atherosclerosis have produced inconsistent findings. The eGFR appeared to be associated with CIMT after adjusting for age and CV risk factors in postmenopausal women [4], hypertensive patients [7], and males with atherosclerotic disease [19]. However, other studies in young and normotensive subjects indicated that eGFR was not associated with CIMT [3-6]. In the current study, we found that the linear association between eGFR and CIMT was likely to be stronger among individuals with higher CV risk. Considering previous and current findings, it is probable that the relationship between eGFR and CIMT depends on individual $\mathrm{CV}$ risk. Associations between urinary albumin excretion and CIMT have been replicated in studies of patients with diabetes [20] or hypertension and impaired glucose tolerance [7]. However, community-based studies have shown inconsistent findings [3, 6]. Age-adjusted albuminuria and CIMT were not associated in a study of Japanese women [3]. In contrast, a study of Koreans showed an independent association between ACR and CIMT even after adjustment for covariates [6]. The current study considered a wide range of covariates and may provide strong evidence supporting the association between urinary albumin excretion and CIMT when our findings are combined with those of the previous Korean study [6].

At present, the underlying pathway linking urinary albumin excretion and GFR with CIMT remains uncertain. One possible model is that kidney dysfunction leads 
to increased deposition of minerals and glycation products, which in turn cause arterial remodeling [21]. Proatherogenic abnormal lipid metabolism, inflammation, disturbances in mineral metabolism and perturbations of macrophages may play roles in atherogenesis [22]. However, atherosclerosis in individuals with CV risk factors may affect kidney function. Because of the cross-sectional design of the current study we were unable to verify whether the decrease in eGFR or the increase in urinary albumin excretion preceded the increase in CIMT or followed it. eGFR and ACR have been found to be significantly heritable in family studies of patients with diabetes or hypertension $[11,12,23]$. The current study confirmed that kidney function and CIMT are also highly heritable in a Korean sample. The heritability of eGFR in the current study was similar to that reported in European subjects with type 2 diabetes mellitus (0.74) [12], but much greater than that reported by the Framingham Heart Study (0.33) [9], the Vietnam Era Twin Registry (about 0.50) [13], African-American siblings with hypertension (0.26) and European-American siblings with hypertension (0.36) [23]. The heritability of ACR estimated in the current study was similar to the range reported by other studies $[10-12,23]$. The heritability of IMT at the CCA segment in our study was comparable to estimates drawn from other populations $[14,24]$, whereas the heritability of IMT at the ICA segment differed from those of other studies, in part due to lower reproducibility related to technically low accessibility [14].

Despite the significant heritability of kidney function and CIMT, evidence supporting a genetic association between CIMT and kidney function was found between eGFR and CIMT. This implied the possibility of genetic pleiotropic action between decreased eGFR and a thickening of CIMT. To our knowledge, there are no comparable previous findings and further studies to replicate the current results are warranted. There were some po- tential limitations in the current study. Although radiolabeled markers such as chromium-51-labeled ethylenediaminetetraacetic acid or nonradioactive contrast media such as iothalamate are ideal methods for determining GFR [25], eGFR was calculated using the Chronic Kidney Disease Epidemiology Collaboration equation, which might have limited precision, even though it is considered to be more accurate than the Modification of Diet in Renal Disease Study equation [18]. We were unable to fully consider the possibility that ACR might be influenced by conditions such as systemic inflammation and microvascular damage [26]. Moreover, the validity of CIMT measurements may have influenced our results. However, considering that biases related to measurements are likely to be random and that the estimations of reliability between CIMT measurements by two different ultrasounds were acceptable, measurement error is unlikely to have affected our study results.

In conclusion, decreased eGFR and increased ACR were significantly associated with CIMT and there was a common genetic correlation between eGFR and CIMT in our Korean twin sample. These findings suggest that eGFR and ACR are potential surrogate markers of carotid atherosclerosis and that further studies are warranted to identify the genetic variants that are shared between CIMT and eGFR.

\section{Acknowledgments}

This study was supported by Inje University (2009), the Korean Centers for Disease Control Research Program (2005-3472400-2440-215, 2006-347-2400-2440-215, 2007-347-2400-2440215, 2008-E00255-00 and 2009-E00500-00), the Korean Ministry of Knowledge Economy (10033321) and the Korea Research Foundation of Ministry of Education, Science and Technology (20100025814 and 20100029113).
References

Kidney Function and Carotid

Intima-Media Thickness
1 Levey AS, Eckardt KU, Tsukamoto Y, Levin A, Coresh J, Rossert J, De Zeeuw D, Hostetter TH, Lameire N, Eknoyan G: Definition and classification of chronic kidney disease: a position statement from Kidney Disease: Improving Global Outcomes (KDIGO). Kidney Int 2005;67:2089-2100.

- 2 Lorenz MW, Markus HS, Bots ML, Rosvall M, Sitzer M: Prediction of clinical cardiovascular events with carotid intima-media thickness: a systematic review and metaanalysis. Circulation 2007;115:459-467.

-3 Ishizaka Y, Ishizaka N, Tani M, Toda A, Toda E, Koike K, Yamakado M: Relationship be- tween albuminuria, low eGFR, and carotid atherosclerosis in Japanese women. Kidney Blood Press Res 2008;31:164-170.

4 Kastarinen $\mathrm{H}$, Ukkola O, Kesaniemi YA: Glomerular filtration rate is related to carotid intima-media thickness in middle-aged adults. Nephrol Dial Transplant 2009;24: 2767-2772.

5 Zhang L, Zhao F, Yang Y, Qi L, Zhang B, Wang F, Wang S, Liu L, Wang H: Association between carotid artery intima-media thickness and early-stage CKD in a Chinese population. Am J Kidney Dis 2007;49:786792. 
6 Choi SW, Kim HY, Lee YH, Ryu SY, Kweon SS, Rhee JA, Choi JS, Shin MH: eGFR is associated with subclinical atherosclerosis independent of albuminuria: the Dong-gu study. Atherosclerosis 2010;212:661-667.

-7 Ishizaka N, Ishizaka Y, Toda E, Koike K, Seki G, Nagai R, Yamakado M: Association between chronic kidney disease and carotid intima-media thickening in individuals with hypertension and impaired glucose metabolism. Hypertens Res 2007;30:1035-1041.

8 Lee M, Saver JL, Chang KH, Liao HW, Chang SC, Ovbiagele B: Low glomerular filtration rate and risk of stroke: meta-analysis. BMJ 2010;341:c4249.

$\checkmark 9$ Fox CS, Yang Q, Cupples LA, Guo CY, Larson MG, Leip EP, Wilson PW, Levy D: Genome-wide linkage analysis to serum creatinine, GFR, and creatinine clearance in a community-based population: the Framingham Heart Study. J Am Soc Nephrol 2004;15: 2457-2461.

10 Fogarty DG, Rich SS, Hanna L, Warram JH, Krolewski AS: Urinary albumin excretion in families with type 2 diabetes is heritable and genetically correlated to blood pressure. Kidney Int 2000;57:250-257.

- 11 Freedman BI, Beck SR, Rich SS, Heiss G, Lewis CE, TurnerS, Province MA, Schwander KL, Arnett DK, Mellen BG: A genome-wide scan for urinary albumin excretion in hypertensive families. Hypertension 2003;42:291296.
12 Langefeld CD, Beck SR, Bowden DW, Rich SS, Wagenknecht LE, Freedman BI: Heritability of GFR and albuminuria in Caucasians with type 2 diabetes mellitus. Am J Kidney Dis 2004;43:796-800.

13 Raggi P, Su S, Karohl C, Veledar E, RojasCampos E, Vaccarino V: Heritability of renal function and inflammatory markers in adult male twins. Am J Nephrol 2010;32:317-323.

14 Moskau S, Golla A, Grothe C, Boes M, Pohl C, Klockgether T: Heritability of carotid artery atherosclerotic lesions: an ultrasound study in 154 families. Stroke 2005;36:5-8.

15 Sung J, Cho SI, Lee K, Ha M, Choi EY, Choi JS, Kim H, Kim J, Hong KS, Kim Y, Yoo KY, Park C, Song YM: Healthy twin: a twin-family study of Korea - protocols and current status. Twin Res Hum Genet 2006;9:844848

16 Song YM, Lee D, Lee MK, Lee K, Lee HJ, Hong EJ, Han B, Sung J: Validity of the zygosity questionnaire and characteristics of zygosity-misdiagnosed twin pairs in the Healthy Twin Study of Korea. Twin Res Hum Genet 2010;13:223-230.

17 Wilson PW, D'Agostino RB, Levy D, Belanger AM, Silbershatz H, Kannel WB: Prediction of coronary heart disease using risk factor categories. Circulation 1998;97:18371847.

18 Levey AS, Stevens LA, Schmid CH, Zhang Y, Castro AF, Feldman HI, Kusek JW, Eggers P, Van Lente F, Greene T, Coresh J, Chronic Kidney Disease Epidemiology Collaboration: A new equation to estimate glomerular filtration rate. Ann Intern Med 2009;150: 604-612.

19 Kawamoto R ON, Kusunoki T, Yorimitsu N An association between the estimated glomerular filtration rate and carotid atherosclerosis. Intern Med 2008;47:391-398.
20 Huang Y, Chen Y, Xu M, Gu W, Bi Y, Li X, Ning G: Low-grade albuminuria is associated with carotid intima-media thickness in Chinese type 2 diabetic patients. J Clin Endocrinol Metab 2010;95:5122-5128.

-21 Peralta CA, Katz R, Madero M, Sarnak M, Kramer H, Criqui MH, Shlipak MG: The differential association of kidney dysfunction with small and large arterial elasticity: the multiethnic study of atherosclerosis. Am J Epidemiol 2009;169:740-748.

22 Yamamoto S, Kon V: Mechanisms for increased cardiovascular disease in chronic kidney dysfunction. Curr Opin Nephrol Hypertens 2009;18:181-188.

23 Leon JM, Freedman BI, Miller MB, North KE, Hunt SC, Eckfeldt JH, Lewis CE, Kraja AT, Djousse L, Arnett DK: Genome scan of glomerular filtration rate and albuminuria: the HyperGEN study. Nephrol Dial Transplant 2007;22:763-771.

-24 Xiang AH, Azen SP, Buchanan TA, Raffel LJ, Tan S, Cheng LS, Diaz J, Toscano E, Quinonnes M, Liu CR, Liu CH, Castellani LW, Hsueh WA, Rotter JI, Hodis HN: Heritability of subclinical atherosclerosis in Latino families ascertained through a hypertensive parent. Arterioscler Thromb Vasc Biol 2002;22: 843-848.

25 Frennby B, Sterner G: Contrast media as markers of GFS. Eur Radiol 2002;12:475484.

26 Nahas ME: Cardio-kidney-damage: a unifying concept. Kidney Int 2010;78:14-18. 